\title{
Data-Driven Engineering - Definitions and Insights from an Industrial Case Study for a New Approach in Technical Product Development
}

\author{
Jakob Trauer ${ }^{1}$, Sebastian Schweigert-Recksiek ${ }^{1}$, Luis Onuma Okamoto ${ }^{1}$, Karsten \\ Spreitzer², Markus Mörtl'1, Markus Zimmermann' $^{1}$ \\ ${ }^{1}$ Laboratory for Product Development and Lightweight Design, Technical University of \\ Munich \\ jakob.trauer@tum.de \\ ${ }^{2}$ Viessmann Werke Allendorf GmbH
}

\begin{abstract}
The growing digitization affects all areas of engineering. Together with fast-paced trends, it drives complexity and uncertainty in many domains. Yet, its potentials are manifold and, in most cases, outweigh the disadvantages. Beneath terms such as "big data", "digital twin", the term "data-driven engineering" has evolved over the last years. However, neither in literature nor in industry, there is a unified definition or understanding of the term. The presented research is based on a literature review as well as an industrial case study. Several databases were screened systematically for the literature review and forward and backward searches were used additionally. The case study was conducted in a collaboration with a company in the climate system sector. First, a literature-based distinction between the terms model-based, modeldriven, data-based, and data-driven as well as definitions of data-driven engineering were investigated. Representatives of the company then evaluated these findings in a workshop and together with the industry partner a consistent definition was developed. The authors define data-driven engineering as a framework for product development in which the goal-oriented collection and use of sufficiently connected product lifecycle data guides and drives decisions and applications in the product development process. Further, promising use cases for the industry partner regarding data-driven engineering were formulated. The use cases were initially evaluated and prioritized regarding their cost-benefit ratio. Symbioses with other strategies of the company such as Digital Twins, model-based engineering, and solution space engineering are outlined. For academia, the presented findings provide a consistent definition that can be used as a promising direction for future research. Especially a procedure model for the systematic conception and implementation of data-driven engineering would be beneficial. For industry, this paper provides insights on potentials of data-driven engineering, a differentiation from related concepts, and very concrete use-cases serving as a starting point for a company-specific implementation.
\end{abstract}

Keywords: data driven design, use-phase data, product development, design process, internet of things (IoT) 


\section{Introduction}

In the course of the digitization of products and processes of companies, more data is created in the entire product life cycle than ever before (Hienz, 2014). This phenomenon is often described as Big Data. Mauro, Greco, and Grimaldi (2016) describe Big Data as "[...] the Information asset characterised by such a High Volume, Velocity and Variety to require specific Technology and Analytical Methods for its transformation into Value.". Thus, this term entails three aspects - the obstacles that occur due to the high complexity of data, the need for special tools and procedures, and the potentials of this trend to create value. In recent years, academia and industry primarily focused on potentials for service development and social media (e.g. Troilo, Luca, \& Guenzi, 2017; Wilberg, Triep, Hollauer, \& Omer, 2017; Zhan, Tan, Li, \& Tse, 2018). However, there are numerous values for technical product development that could be achieved by analysing data generated over the entire product lifecycle (Hienz, 2014; Zhan et al., 2018). According to Eckert et al. (2019), over the next decades ,all“" products will be cyberphysical systems. In addition, product development will be affected by the increasing interconnectivity of these product, providing analyses to drive design decisions (Eckert et al., 2019; Spath \& Dangelmaier, 2016). Multiple use cases and approaches to support this paradigm shift are already known such as Digital Twins (Spath \& Dangelmaier, 2016; Trauer, Schweigert-Recksiek, Engel, Spreitzer, \& Zimmermann, 2020). Yet, there is still no unified definition, framework, or understanding of what could be described as "data-driven engineering" (DDE). Consequently, the goal of this publication is to present an overarching definition of DDE, which can be used to classify novel approaches, but also to guide the previously described paradigm shift, supporting companies to compete in such complex and fast-paced markets.

\section{Methodology}

\subsection{Industrial Case-Study}

The industrial case study partner is Viessmann, a medium-sized company with more than 10,000 employees. The company develops and designs climate systems for residential and commercial applications. As many other companies, on the one hand the case study partner strives to profit from the high potentials of the ongoing digitization, but on the other hand has to face the challenges of the ambitious transition and the negative effects of an increasingly complex environment. To overcome these threads, the company is undergoing an extensive change process. Some first steps have already been taken, as more and more connectivity services are included in the products. However, their purpose is primarily to increase the customer satisfaction directly by providing possibilities e.g. to control the climate solution via app. Nevertheless, more engineering-oriented approaches are in the planning, such as the introduction of a Digital Twin (Schweigert-Recksiek, Trauer, Engel, Spreitzer, \& Zimmermann, 2020).

\subsection{Systematic Literature Review}

The research presented in this paper is based on the Design Research Methodology of Blessing and Chakrabarti (2009). The impression of the literature-based research clarification indicated an insufficient understanding of DDE. Consequently, type 2 of the research methodology "Comprehensive Study of the Existing Situation" - was applied. Based on the research clarification, a comprehensive descriptive study was conducted analysing the state of research in literature, followed by an initial workshop-based prescriptive study. Two research questions (RQ) guided the systematic literature review: 
- RQ1 What are the key characteristics of DDE?

- RQ2 Where are the differences between DDE and other existing approaches?

Three search mechanisms delivered the results of the literature review. First, a keyword search process was conducted. A research strategy was built to structure and guide the systematic search process (cf. Figure 1).

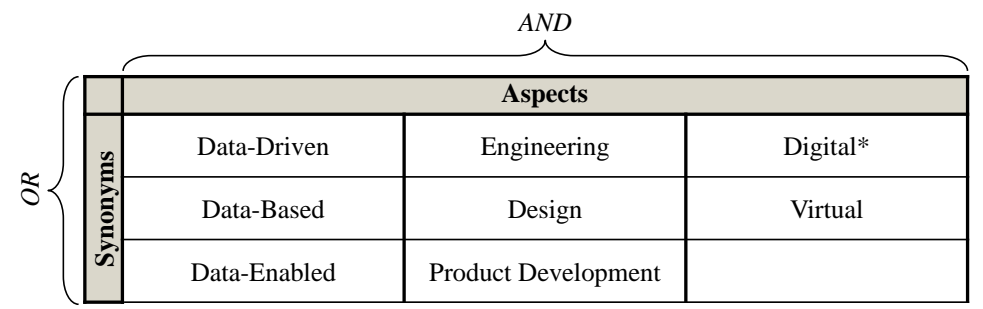

Figure 1. Literature Research Strategy

Considering only "data-driven engineering" as search phrase, 24 results are indicated. In the context of engineering there are only 14, twelve of which use it just once, or as a keyword. The remaining two publications are in the field of biotechnology and thus are not in the scope of this paper of mechatronic engineering. Therefore, the previously mentioned keywords were used. Combining these keywords using Boolean operators as indicated in Figure 1 delivered the search phrase. This phrase was entered in Scopus. Consequently, the results were filtered down to 92 publications, as shown in Figure 2.

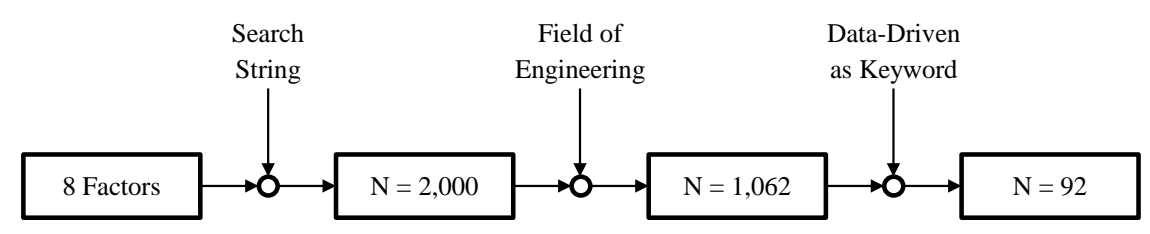

Figure 2. Keyword Search Process

The results of the keyword search process were accompanied by a targeted consultation of textbooks, journals, and websites. Further, interesting references from identified literature were traced back. Overall, the authors investigated 119 publications. The most relevant publications are presented in the following section.

\section{Literature Review}

\subsection{Distinction of Basic Terms}

The adjective "data-driven" occurs in combination with many, very different terms in literature in highly divergent topics. What does it actually mean? Often it is used synonymously with the adjective "data-based" and in combination with adjectives such as "model-based", or "modeldriven". What is the distinction between those terms? Consequently, as a first step, those basic questions need to be clarified.

The ISO International Organization for Standardization (2015) defines data as "reinterpretable representation of information in a formalized manner suitable for communication, interpretation, or processing”. Many publications equate data and information. However, data is just a representation of information. Only if the context is known, it can be interpreted and information is extracted (Bauer \& Dangelmaier, 2016). For data-based or data-driven approaches, especially data from after-sales phases of a product are of interest. According to Wilberg et al. (2017), for this kind of data, the term use-phase data is used. Models are used in 
all fields of engineering and design (Eckert \& Hillerbrand, 2019). According to Lindemann (2009), models are, compared to the original, a simplified mental or physical object to draw purpose-oriented analogies and conclusions about the real object. There is a plethora of different models. process and product models are especially in engineering relevant, representing specific aspects of the process or product in a simplified manner (Eckert \& Hillerbrand, 2019; Lindemann, 2009). Often models use data as an input. Thus, they can be used to decode the entailed information of data. Consequently, information is formalized by data to enable communication, processing, and interpretation, which can be done using models.

The distinction between "-based" and "-driven" is more complicated. According to Cambridge University Press (2020), the suffix "-based" indicates that something is developed from the prefix. "-driven" instead means that something is caused or influenced by something or to make something happen (Cambridge University Press, 2020). Whereas the former suffix is passive, the latter indicates an active influence. This is valid for example in software engineering. Here, "Model-Based Software Engineering" entails just the systematic use of models as primary engineering artefact. In "Model-Driven Software Engineering" instead, models are (semi-) automatically transferred into other models, or (semi-)automatically generate code by themselves (Nyßen, 2009). Regarding the terms data-based and data-driven no concrete differentiation was found in literature. King, Churchill, and Tan (2017) introduce in addition to data-driven, the terms data-informed and data-aware. In data-informed design, decisions are prepared with insights from data, but other sources of information also affect the decision. In a data-aware mindset the decision maker is not limited to data. It is rather about to be aware that there are many sources to solve a problem and the best one is chosen, which does not have to be data. Considering the definition of the adverb "-based", the two additionally suggested terms can be taken as subsets of "data-based". There are several publications using "data-based" and "data-driven" in combination with other terms, which will be presented in the following section.

\subsection{Related Methods and Frameworks}

From the literature review no consistent definition of the adjective data-driven was found. Literature in the context of engineering is also sparse. However, literature provides some related frameworks, procedure models, and methods that are indicating characteristics of DDE. These publications are presented in the following.

In the context of computation and machine learning, especially the terms "Data-Driven Modelling" or "Data-Driven models" appear. The former is an approach analysing data of a system in order to model dependencies between system variables without exactly knowing the physical relations (D. Solomatine, See, \& Abrahart, 2008). This is especially required for very complex products and can be an interesting use case in engineering as well, e.g. for solutionspace engineering (Zimmermann \& Hoessle, 2013). "Data-Driven Models" is a related technique. It can be used predictive or descriptive - either to predict values of variables, or to reveal and interpret patterns in data (Anand \& Büchner, 1998).

The latter application scenario can often be found in the field of data science and increasingly finds its way into engineering. In this field, decisions often rely on data. An example is "DataDriven Decision Making". Here, data is gathered in order to support the decision-making. Thus, decisions are expected to become more rational and less influenced by gut instincts (Brynjolfsson, Hitt, \& Kim, 2011). There is also evidence of the positive influences of this technique (e.g. Provost \& Fawcett, 2013).

In most cases, these methods are used for market analyses, or requirements engineering. Consequently, data-driven concepts often focus on customers - for instance "Cyber-Empathic Design". Ghosh, Olewnik, Lewis, Kim, and Lakshmanan (2017) describe it as a framework to integrate data of user-product interactions into design activities. Thus, individual customer 
perceptions and preferences can be tracked from the use phase using embedded sensors to drive innovations and product portfolio decisions. A related framework is "Data-Driven Product Design" with the difference that the focus is explicitly on the perception of a products visual design (Chien, Kerh, Lin, \& Yu, 2016). This term can be used interchangeable with "Product Design Analytics" (Ma, Kwak, \& Kim, 2014). In another use case based on these frameworks, product families can be designed in a data-driven way - which is called "Predictive DataDriven Product Family Design" (Ma \& Kim, 2016). Zheng, Feng, Gao, and Tan (2018) describe these applications of product and use-phase in product development as the next paradigm shift in engineering. Nowadays, instead of relying merely on expert knowledge, "Data-Driven Product Development" can be used to derive good, robust, and feasible designs, driven by product data (Zheng et al., 2018).

Bogers, Frens, van Kollenburg, Deckers, and Hummels (2016) present a framework, called "Data-Enabled Design", for the integration of use phase data in the design of a product-service system. They integrated real-time use phase data as well as user interactions to iteratively improve the product at hand. They also differentiate data-enabled from data driven - datadriven approaches use much larger data sets (big data) but less qualitative insights to contextualize the data (Bogers et al., 2016).

A state-of-the-art approach in engineering is model-based systems engineering. As indicated by the name, it is based upon models. Guardabasso, Lindblad, Witzmann, and Siarov (2019) however propose a "Data-Driven Systems Engineering" methodology in order to improve the traditional model-based procedure. By "data-driven" Guardabasso et al. (2019) mean the consistent integration of engineering design data and its connections into the systems engineering process. This approach is based on a single source of truth and seems to be closely related to the concept of a digital thread, or digital twin. In this approach, the focus is on engineering design data, not on use-phase data.

Another term that appears quite often in literature, especially in the field of decision-making user experience and integration (UX/UI) is "Data-Driven Design". It is a much more established research field than most of the approaches stated above. When searching for this term on Scopus, 245 results are indicated. According to King et al. (2017), in data-driven design decisions (especially in the context of user experience) are guided by the collected data. Liu and Chen (2017) for example describe a data-driven design paradigm as a framework for decision-making, where the decision is formulated as a mathematical model and can be optimised. Kusiak (2006) presents different applications of data-driven design, where by linking data to decision making product innovations are driven. In most of the described application scenarios the analysed data led to decision, innovations, or product and process improvements.

\section{Results}

\subsection{Definition of Data-Driven Engineering}

The previous section presented related terms, concepts, and definitions of DDE based on literature. These findings were discussed also with five engineers from the industry partner in order to come up with a consistent definition for DDE.

First, the adjective data-driven was discussed. The authors and the industry partner do see a difference between data-based and data-driven. When for example a decision is made databased, data was just used to support, or to validate the human decision. However, when it is made data-driven, an automated interpretation of the underlying data can prepare and indicate a decision, which only needs to be approved by humans. 
To make this clearer, an analogy was built on navigating in a car. In the basic version, the driver would use a paper map, investigate a feasible route, and then navigate according to it. Considering the map as data, he would only base all his decisions on the available data, but it would need some effort to analyse it and it would not be given that it is the best (fastest, most beautiful, shortest, etc.) route. The more elaborate version would be a data-driven system. Consider a navigation system such as Google Maps or Apple Maps, integrating a map of traffic data etc. With the underlying algorithms, the system itself can analyse the data and find the best insights - i.e. a route that is driven by the data to fulfil the requirements as well as possible. However, there is still a person driving the car. The driver just does not need to make a decision on the route. Consequently, data-based approaches are more "reactive". When a decision needs to be made, data is analysed. Data-driven instead is "proactive". Proactively data is included and implemented in the engineering process. Underlying models and algorithms deliver directions for innovations or decisions more or less automatically. With this basic understanding in mind a definition for DDE was developed:

Data-driven engineering is a framework for technical product development in which the usecase-oriented collection and utilization of sufficiently connected product lifecycle data guides and drives decisions and applications in the product development process.

The elements of the definition are described in the following to elaborate its characteristics. The authors consider DDE to be a framework. Thus, it should include processes, methods, tools, roles, and context factors, which support the implementation of DDE.

There should be a use-case-oriented gathering of data. Consequently, only required data points in the right quality and the right format are transmitted and analysed. This is a prerequisite for this framework and in line with other approaches, for example with the use-phase data strategy of (Wilberg, Fahrmeier, Hollauer, \& Omer, 2018). Thus, this is a central characteristic of DDE. To achieve all potentials of this framework, engineers need to include the right technologies and sensor in the products developed today in order to gather data tomorrow that is used to improve the product development of future products. This is a new paradigm in engineering and influences most parts of the product development process.

How the gathered data can be used is manifold. Use cases can range from predictive maintenance, over production planning or innovation management to organizational optimizations of the engineering process. The definition seems to be very ambitious and rigid. Here, the authors suggest a model (based on Deshpande, Sharma, \& Peddoju, 2019) as shown in Figure 3. Starting with use cases of descriptive analytics DDE can be achieved stepwise by elaborating the use cases further towards prescriptive analytics. Consequently, DDE is more like a vision, which is formulated as: With data-driven engineering, decisions are made in every phase of the product development process through the goal-oriented collection and use of data from the entire product life cycle. Data is not just the basis of decision but drives the decisionmaking and innovation processes through underlying analyses.

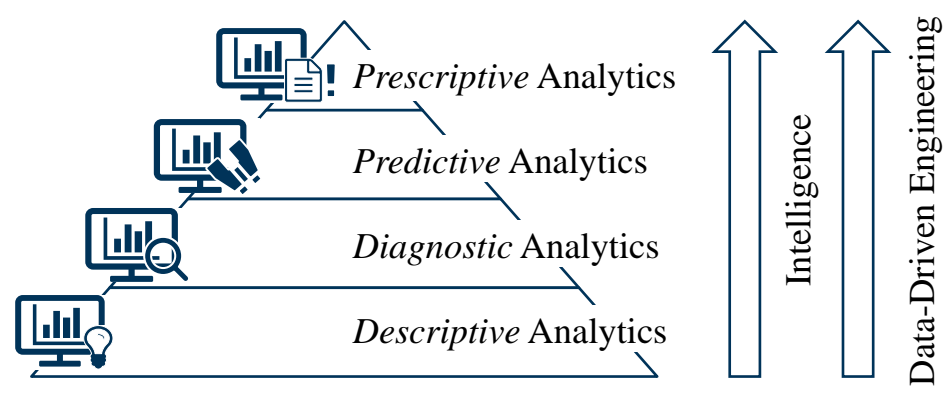

Figure 3. Levels of Data Analytics towards DDE (based on Deshpande et al., 2019) 
In order to use the data, it needs to be connected sufficiently. In the highest level of DDE, data from all phases of a product's lifecycle should be used. Thus, the product lifecycle circle can be closed and already produced and sold products deliver data that can be used to design the products of the next generation. To do so, they need to be connected. However, connecting all data would be a utopia. Consequently, data need to be connected sufficiently to achieve the envisaged use case or vision. Therefore, a strong enabler of DDE could be the concept of a digital twin, entailing information, models, and data from all phases of the lifecycle (Trauer et al., 2020).

The most important characteristic is that decisions and applications in the product development process can be driven by data - not only based on but also driven. For example, in an automated way, requirements can be updated, or concept decisions can be made. Instead of just validating or strengthening the gut feeling of an engineer regarding a decision, the system itself would give a suggestion, which then needs to be checked by the engineer. Not only the subjective bias of experts is reduced - decisions depend not only on the current state of information, but also on potential future information. With a data-driven approach, predictions can be made on a rational basis and updated regularly.

To conclude, the following values of data-driven are suggested. While the other side is still important, DDE should put emphasis on:

- Prescriptive over descriptive approaches,

- Proactive over reactive approaches,

- Dynamic over static data,

- Real-time over historic data.

\subsection{Use Cases at the Industry Partner}

To elaborate the definition further and to find a starting point for the further development of DDE, promising use cases were developed in a four-hour long workshop together with the industry partner. First, in a mute brainstorming session the attendants filled out use case templates consisting of a title, a user story, a problem description, expected value, and expected effort of the use case. Further, contextual information on product, data source etc. were collected. Next, each representative of the company presented their use cases to the others, before the attendees evaluated the use cases qualitatively regarding value and effort on a scale from one to five. The rating resulted in the following portfolio, depicted in Figure 4. The portfolio contains the averages of the attendee's voting.

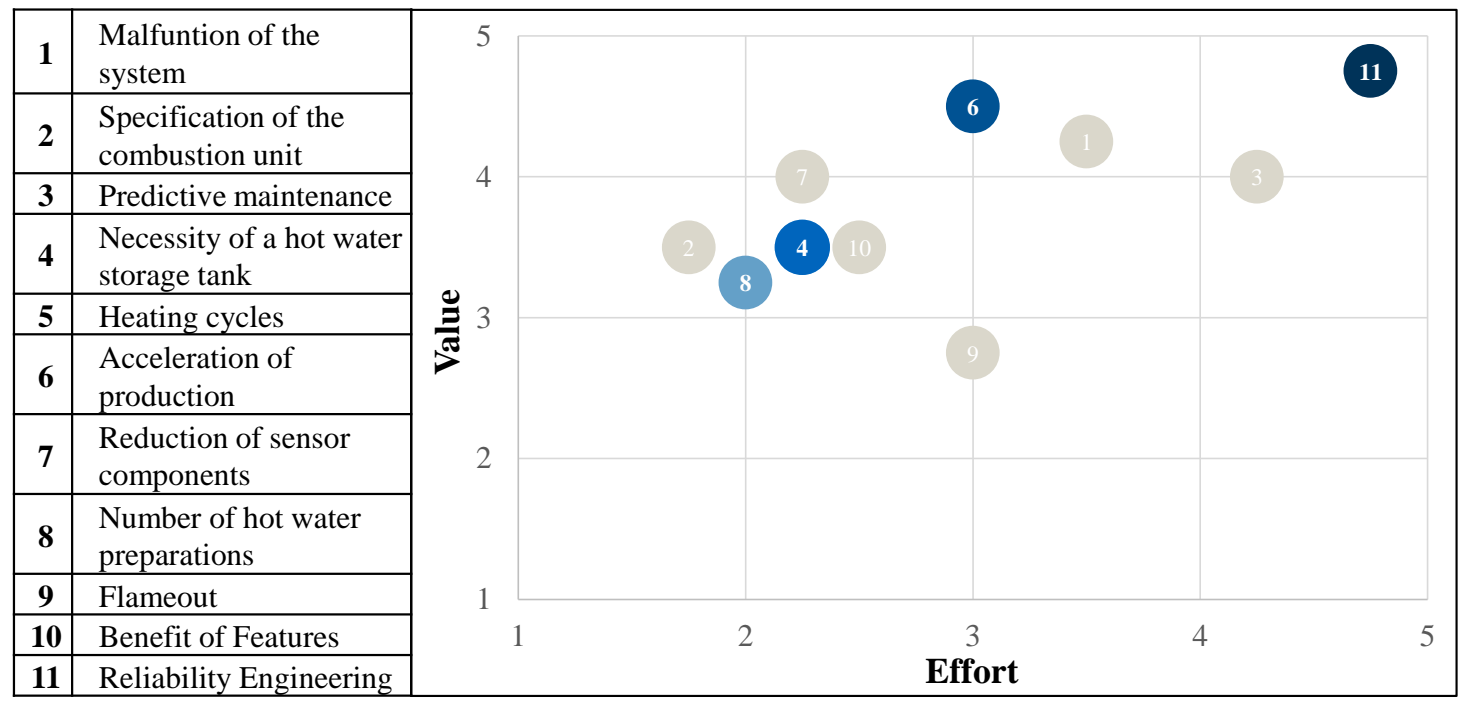

Figure 4. Evaluation of Use Cases Related to Data-Driven-Engineering 
With this portfolio at hand, it was possible to select the four most promising use cases - namely use case 4, 6, 8, and 11 . Use case 8 regards heat pumps. Heat pumps have a valve that directs the hot water from the heat pump either into the house for heating or into the hot water tank for drinking water. Currently, there are frequent hot water preparations, the cause of which are unclear. A possible cause could be a leaking valve or simply a high house consumption of water. So far, the reason is not exactly known. With use phase data of the whole heat pump and more specifically of the valve, it would be possible to identify the real cause for this issue. The analysed data could indicate a new specification for the valve, or adjusted requirements for the whole system. With this use case it would be possible to improve the efficiency of the climate system, increase the customer satisfaction, and reduce maintenance costs. The effort was rated medium to low, as some of the contextual data is already gathered by service systems.

Use case 4 also tackles a problem for heat pumps. In order to be able to make currently unused heat from a heat pump available on demand at a later date, a buffer tank is positioned between the heat generator and the heat sink. This is also important as it decreases the need of frequent compressor launches which increases the compressor's lifetime. However, with novel compressor technologies, a hot water tank as storage might not be needed anymore. To prove this hypothesis, just data from the compressor would be needed. Some of this data is already collected and the analysis would be relatively easy, so the effort was rated low. As it would be possible to save some components and thus reduce costs, the value was rated medium to high. Use case 6 deals with the reduction of production and maintenance time. With the specific (de-) assembly times from production and service, it would be possible to indicate potentials for improvement for the components within the heating system. A model could be filled with that data resulting in concrete hints for improvement in the design of the components. On the one hand, the gathering of data would be relatively easy, however, building a model to analyse the data might be more complicated. Thus, the effort was rated medium. On the other hand, the value would be quite high - it could be possible to reduce production and maintenance time, reduce costs, and improve the efficiency of the production line.

Use case 11 is the most ambitious but also most promising one. It is difficult to predict how the components will be stressed in the field. Thus, the component designs are subject to great uncertainty, for example with regard to the collectives of loads. This problem is even worse for supplier parts, as there is also uncertainty as to whether the components have been developed and tested in accordance with the requirements. Therefore, if use-phase data on these load collectives would be available already in the design phase, specifications could be formulated more accurate, the components fit for intended use could be increased and the reliability engineering would be improved. To achieve this goal elaborate analyses and a huge amount of data would be required. Thus, the effort was rated as high. However, this use case can be achieved stepwise by implementing smaller use cases such as use case 4 and 8 , which directly contribute to this goal.

\section{Conclusion}

\subsection{Discussion}

This paper presented a definition of DDE. Therefore, related literature was analysed and discussed with the industry partner. It was hard to draw the line on potentially interesting related literature. Thus, there might be aspects, which might not have been addressed. The selection was made together with the industry partner. The intention of this paper was not to develop a completely new concept. The perception was rather, that there is an ongoing paradigm shift in engineering, yet there is no term or description for it. Consequently, many publications presented in section 3 do not stand in contradiction to our definition but can be seen as a subset 
of it. The presented definition was also evaluated with four representatives of the industry partner (cf. Figure 5). To assure that the attendees understood the definition, also the understanding was evaluated. The results were documented in a portfolio. Each small circle represents the rating of one participant. The big dot in the middle is the average score. In total, the understanding was quite high. The overall relevance was rated as high as well and especially for their company as a promising new approach.

Data-Driven Engineering

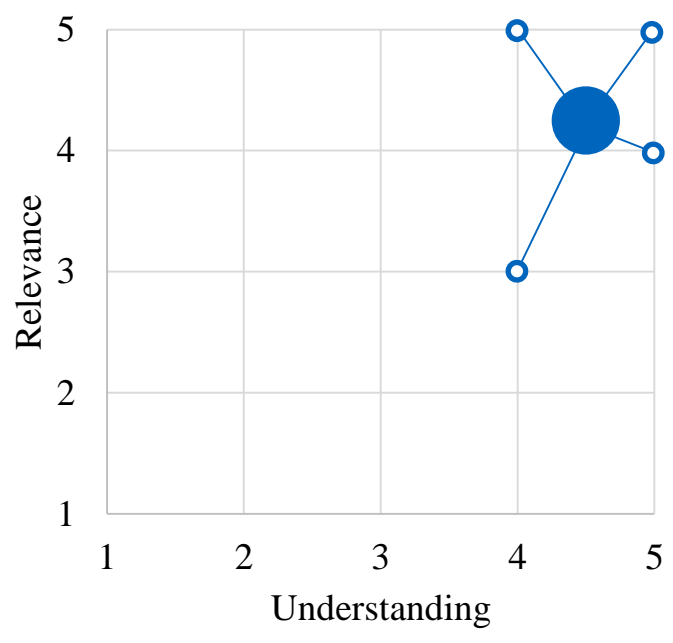

Figure 5. Evaluation of the Definition of DDE

However, there are also some limitations to overcome. The biggest limitation was seen in the use-case oriented collection of data. It seems hard to evaluate potential use cases and specially to assess their business case in order to get the approval to implement required technologies to achieve the use case. Further, before implementing use cases the basic conditions need to be assured, e.g. a consistent data format, software tools for the analyses, etc. Further, the definition was perceived as quite strict and ambitious. However as already stated earlier, it should serve as a vision - as an ambitious goal that can be achieved step by step through smaller projects. In terms of the vision, there are of course further limitations specially as innovation an decision processes depend on a plethora of other factors, which makes it harder fully cover these aspects with DDE.

\subsection{Outlook}

In the future collaboration a procedure model for the implementation of DDE will be developed. This will be done based on the use cases presented in section 4.2. Combining our approach with the use-phase data strategy of Wilberg et al. (2018) should help to overcome the challenge of the use-case oriented collection of data. To evaluate the potential use cases, also methods and approaches to develop valid business cases will be investigated. This project will be connected with an ongoing project on digital twins (Schweigert-Recksiek et al., 2020) as well as an project on solution space engineering.

\section{Acknowledgments}

The authors thank Viessmann for the very fruitful collaboration. 


\section{References}

Anand, S. S., \& Büchner, A. G. (1998). Decision support using data mining. Management briefings. Information technology. London: Financial Times Management.

Bauer, W., \& Dangelmaier, M. (2016). Virtuelles Engineering. In U. Lindemann (Ed.), Handbuch Produktentwicklung (pp. 939-978). Munich: CARL HANSER Verlag GMBH $\&$.

Blessing, L. T.M., \& Chakrabarti, A. (2009). DRM, a Design Research Methodology. London: Springer London. https://doi.org/10.1007/978-1-84882-587-1

Bogers, S., Frens, J., van Kollenburg, J., Deckers, E., \& Hummels, C. (2016). Connected Baby Bottle. In A. S. I. G. o. C.-H.M. Interaction (Ed.), Proceedings of the 2016 ACM Conference on Designing Interactive Systems (pp. 301-311). [Place of publication not identified]: ACM. https://doi.org/10.1145/2901790.2901855

Brynjolfsson, E., Hitt, L. M., \& Kim, H. H. (2011). Strength in Numbers: How Does DataDriven Decisionmaking Affect Firm Performance? SSRN Electronic Journal. Advance online publication. https://doi.org/10.2139/ssrn.1819486

Cambridge University Press (2020). Cambridge Dictionary. Retrieved from https://www.dictionary.cambridge.org/

Chien, C.-F., Kerh, R., Lin, K.-Y., \& Yu, A. P.-I. (2016). Data-driven innovation to capture user-experience product design: An empirical study for notebook visual aesthetics design. Computers \& Industrial Engineering, 99, 162-173. https://doi.org/10.1016/j.cie.2016.07.006

Deshpande, P. S., Sharma, S. C., \& Peddoju, S. K. (2019). Predictive and Prescriptive Analytics in Big-data Era. In P. S. Deshpande, S. C. Sharma, \& S. K. Peddoju (Eds.), Studies in Big Data: Vol. 52. Security and Data Storage Aspect in Cloud Computing (Vol. 52, pp. 71-81). Singapore: Springer Singapore. https://doi.org/10.1007/978-981-13-60893 -5

Eckert, C., \& Hillerbrand, R. (2019). Models in Engineering Design: Generative and Epistemic Function of Product Models. In P. E. Vermaas \& S. Vial (Eds.), Design Research Foundations. Advancements in the Philosophy of Design (Vol. 17, pp. 219-242). Cham, Switzerland: Springer International Publishing. https://doi.org/10.1007/978-3-31973302-9_11

Eckert, C., Isaksson, O., Hallstedt, S., Malmqvist, J., Öhrwall Rönnbäck, A., \& Panarotto, M. (2019). Industry Trends to 2040. Proceedings of the Design Society: International Conference on Engineering Design, 1(1), 2121-2128. https://doi.org/10.1017/dsi.2019.218

Ghosh, D., Olewnik, A., Lewis, K., Kim, J., \& Lakshmanan, A. (2017). Cyber-Empathic Design: A Data-Driven Framework for Product Design. Journal of Mechanical Design, 139(9), 463. https://doi.org/10.1115/1.4036780

Guardabasso, P., Lindblad, L., Witzmann, M., \& Siarov, S. (2019). Evaluation of the Learning Process of a Data-Driven Systems Engineering Methodology in a Workshop Environment. 70th International Astronautical Congress.

Hienz, J. (2014). Defining the Data Movement. In The Future of Data-Driven Innovation: U.S. Chamber of Commerce Foundation.

ISO International Organization for Standardization (2015). Information technology Vocabulary. (Standard, ISO/IEC 2382:2015(en)).

King, R., Churchill, E. F., \& Tan, C. (2017). Designing with data: Improving the user experience with $A / B$ testing (First edition). Sebastopol, CA: O'Reilly. 
Kusiak, A. (2006). Data mining: manufacturing and service applications. International Journal of Production Research, 44(18-19), 4175-4191. https://doi.org/10.1080/00207540600632216

Lindemann, U. (2009). Methodische Entwicklung technischer Produkte. Berlin, Heidelberg: Springer Berlin Heidelberg. https://doi.org/10.1007/978-3-642-01423-9

Liu, C., \& Chen, X. (2017). Data-driven design paradigm in engineering problems. Proceedings of the Institution of Mechanical Engineers, Part G: Journal of Aerospace Engineering, 231(8), 1522-1534. https://doi.org/10.1177/0954410016653502

Ma, J., \& Kim, H. M. (2016). Product family architecture design with predictive, data-driven product family design method. Research in Engineering Design, 27(1), 5-21. https://doi.org/10.1007/s00163-015-0201-4

Ma, J., Kwak, M., \& Kim, H. M. (2014). Demand Trend Mining for Predictive Life Cycle Design. Journal of Cleaner Production, 68, 189-199. https://doi.org/10.1016/j.jclepro.2014.01.026

Mauro, A. de, Greco, M., \& Grimaldi, M. (2016). A formal definition of Big Data based on its essential features. Library Review, 65(3), 122-135. https://doi.org/10.1108/LR-06-20150061

Nyßen, A. (2009). Model-based construction of embedded and real-time software: a methodology for small devices. PhD Thesis. Aachener Informatik-Berichte. Aachen: RWTH Aachen, Department of Computer Science.

Provost, F., \& Fawcett, T. (2013). Data science and its relationship to big data and data-driven decision making. Big Data, 1(1), 51-59.

Schweigert-Recksiek, S., Trauer, J., Engel, C., Spreitzer, K., \& Zimmermann, M. (2020). Conception of a Digital Twin in Mechical Engineering: A Case Study in Technical Product Development. Accepted at DESIGN2020. ISSN: 2633-7762

Solomatine, D. [D.], See, L. M. [L. M.], \& Abrahart, R. J. [R. J.] (2008). Data-Driven Modelling: Concepts, Approaches and Experiences. In D. P. Solomatine, R. J. Abrahart, \& L. M. See (Eds.), Practical Hydroinformatics: Computational Intelligence and Technological Developments in Water Applications (1st ed., Vol. 68, pp. 17-30). s.1.: Springer-Verlag. https://doi.org/10.1007/978-3-540-79881-1_2

Spath, D., \& Dangelmaier, M. (2016). Produktentwicklung Quo Vadis. In U. Lindemann (Ed.), Handbuch Produktentwicklung. Munich: CARL HANSER Verlag GMBH \&.

Trauer, J., Schweigert-Recksiek, S., Engel, C., Spreitzer, K., \& Zimmermann, M. (2020). What is a Digital Twin?: Definitions and Insights from an Industrial Case Study in Technical Product Development. Accepted at DESIGN 2020. ISSN: 2633-7762

Troilo, G., Luca, L. M. de, \& Guenzi, P. (2017). Linking Data-Rich Environments with Service Innovation in Incumbent Firms: A Conceptual Framework and Research Propositions. Journal of Product Innovation Management, 34(5), 617-639. https://doi.org/10.1111/jpim.12395

Wilberg, J., Fahrmeier, L., Hollauer, C., \& Omer, M. (2018). DERIVING A USE PHASE DATA STRATEGY FOR CONNECTED PRODUCTS: A PROCESS MODEL. In Design Conference Proceedings, Proceedings of the DESIGN 2018 15th International Design Conference (pp. 1441-1452). University of Zagreb, Croatia; The Design Society, Glasgow, UK. https://doi.org/10.21278/idc.2018.0213

Wilberg, J., Triep, I., Hollauer, C., \& Omer, M. (2017). Big Data in Product Development: Need for a Data Strategy. In D. F. Kocaoglu \& PICMET (Eds.), Technology management for the interconnected world: PICMET '17 : Portland International Conference on 
Management of Engineering and Technology (pp. 1-10). [Piscataway, NJ]: IEEE. https://doi.org/10.23919/PICMET.2017.8125460

Zhan, Y., Tan, K. H., Li, Y., \& Tse, Y. K. (2018). Unlocking the power of big data in new product development. Annals of Operations Research, 270(1-2), 577-595. https://doi.org/10.1007/s10479-016-2379-х

Zheng, H., Feng, Y., Gao, Y., \& Tan, J. (2018). A Robust Predicted Performance Analysis Approach for Data-Driven Product Development in the Industrial Internet of Things. Sensors (Basel, Switzerland), 18(9). https://doi.org/10.3390/s18092871

Zimmermann, M., \& Hoessle, J. E. von (2013). Computing solution spaces for robust design. International Journal for Numerical Methods in Engineering, 94(3), 290-307. https://doi.org/10.1002/nme.4450 